\title{
Repercussões da Menopausa para a Sexualidade de Idosas: Revisão Integrativa da Literatura
}

\author{
Izabella Lenza Crema \\ Rafael De Tilio \\ Universidade Federal do Triângulo Mineiro, MG, Brasil. $\quad$ Universidade Federal do Triângulo Mineiro, MG, Brasil. \\ Maria Teresa de Assis Campos \\ Universidade Federal do Triângulo Mineiro, MG, Brasil.
}

Resumo: A menopausa representa transformações hormonais significativas que podem ser acompanhadas de mudanças sociais e emocionais. Além disso, a população idosa feminina tem apresentado maiores comprometimentos em relação à sexualidade do que os homens. Este estudo teve por objetivo apresentar uma revisão integrativa da literatura científica nacional e internacional sobre as possíveis repercussões da menopausa para a sexualidade de idosas. As buscas foram realizadas nas bases Lilacs, SciELO, PePSIC e PsycINFO com análise da produção científica nacional e internacional relativa ao período de janeiro de 2006 a março de 2016. Foram analisadas 36 produções na íntegra cujo perfil predominante é de estudos quantitativos, descritivos e transversais, desenvolvidos com mulheres de diferentes faixas etárias na pós-menopausa, incluindo idosas. Todavia, estudos com amostras compostas exclusivamente por idosas são minoria. Entre os resultados houve predominância de pesquisas voltadas para a avaliação e quantificação das possíveis patologias e sintomas biológicos que afetam a sexualidade (redução da libido, de lubrificação e de orgasmos, por exemplo). A maioria das pesquisas aponta para a importância da análise conjunta de aspectos biológicos, psicológicos, sociais, culturais e individuais para a compreensão das suas distintas influências na sexualidade das idosas. Destaca-se a necessidade de realização de pesquisas com abordagem mista e voltadas apenas para mulheres idosas a fim de ampliar a compreensão acerca das suas perspectivas sobre as repercussões da menopausa para a sexualidade pretendendo respaldar práticas clínicas e políticas sociais destinadas a essa população.

Palavras-chave: Menopausa, Sexualidade, Climatério, Envelhecimento, Mulher.

\section{Menopause Effects for the Sexuality of Older Adults: An Integrative Literature Review}

\begin{abstract}
Menopause involves hormonal transformations that may be accompanied by social and emotional changes. Besides, female elderly population has more sexual problems than males. This research aimed to present a national and international scientific integrative review about the effects of menopause for elderly women sexuality. The index bases utilized were Lilacs, SciELO, PePSIC and PsycINFO from January 2006 to March 2016. 36 papers were analyzed; most of them were quantitative, descriptive and transversal studies developed with postmenopausal women including elderly woman. However, research exclusively focused to elder women was minority. The mains results point that the productions focused on sexual symptoms and sexual pathologies assessments and quantification (as reduced libido, lubrication and orgasm); these productions also point out the importance of broad analyses that include the biological, psychological, social, cultural and personals aspects of elder women sexuality. We highlight the need for more research with mixed approaches exclusively to elder women to broaden the comprehension of the repercussions of menopause to sexuality, endorsing clinical practice and social policies to this population.
\end{abstract}

Keywords: Menopause, Sexuality, Climacteric, Aging, Woman. 


\title{
Efectos de la Menopausia para la Sexualidad: Una Revisión de Literatura
}

\begin{abstract}
Resumen: La menopausia representa transformaciones hormonales significativas que pueden ser acompañadas de cambios sociales y emocionales. La población senil femenina ha presentado mayores impactos en su sexualidad al ser comparada a la masculina. Este estudio tuvo por objetivo presentar una revisión integrativa de la literatura científica nacional e internacional sobre las posibles repercusiones de la menopausia para la sexualidad de mujeres seniles. Las búsquedas fueron realizadas en Lilacs, SciELO, PePSIC y PsycINFO con análisis de la producción científica nacional e internacional de enero de 2006 a marzo de 2016. Fueron analizados 36 artículos científicos en la íntegra, cuyo perfil predominante es de estudios cuantitativos, descriptivos y transversales desarrollados con mujeres de diferentes grupos de edad en la posmenopausia, incluyendo mujeres seniles. Sin embargo, estudios con muestras compuestas exclusivamente por mujeres seniles son una minoría. Entre los resultados hubo predominancia de estudios de evaluación y calificación de las posibles patologías y síntomas biológicos que afectan la sexualidad (reducción de libido, lubricación y orgasmos, por ejemplo). La mayoría de los estudios apunta para la importancia de un análisis conjunto de aspectos biológicos, psicológicos, sociales, culturales y personales para la comprensión de la sexualidad de las mujeres seniles. Se destaca la necesidad de nuevas investigaciones con abordaje mixto y enfocadas en las mujeres seniles a fin de ampliar la comprensión acerca de la perspectiva de estas sobre las repercusiones de la menopausia en la sexualidad, con el objetivo de respaldar prácticas clínicas y políticas sociales destinadas a esa población.
\end{abstract}

Palabras clave: Menopausia, Sexualidad, Climaterio, Envejecimiento, Mujer.

\section{Introdução}

O Estatuto do Idoso considera idosas pessoas com idade a partir de 60 anos (Brasil, 2003). Nas últimas décadas constata-se uma feminização da velhice: as mulheres representam $55,5 \%$ da população brasileira a partir de 60 anos e $61 \%$ dos idosos acima de 80 anos (IBGE, 2011). Para Bandeira, Melo e Pinheiro (2010), isso seria justificado devido ao aumento da expectativa de vida das mulheres (em média oito anos a mais que os homens), à diferencial exposição aos fatores de risco de mortalidade e à maior adesão aos cuidados de saúde quando comparadas aos homens.

No entanto, observa-se que a qualidade do envelhecimento feminino no Brasil tem sido comprometida devido às desigualdades e desvantagens as quais as mulheres são expostas ao longo da vida tanto nos ambientes familiares como nos profissionais. Nesse sentido, a diferença salarial e a dupla jornada de trabalho podem acarretar às mulheres dificuldades sociais e de saúde ao longo do envelhecimento (Fernandes, 2009). Desse modo, evidencia-se a importância de atenção específica e integral a essa população visando suas demandas e necessidades, dentre elas as relacio- nadas à menopausa e suas implicações para a sexualidade feminina.

Para Araújo, Queiroz, Moura e Penna (2013), a sexualidade está presente em todas as etapas do ciclo vital e é reelaborada durante a vida a partir de influências do contexto social, cultural, econômico, religioso, educacional e psicológico de cada indivíduo. Estes autores pontuam que a sexualidade envolve atributos biológicos (genética, necessidades instintivas e reprodutivas) e subjetivos (afeto, amor, carinho, desejo e estética), apresentando particularidades em cada etapa da vida.

Para Barreto e Heloani (2011), a sexualidade não se limita às relações sexuais (coito) e envolve gestos, atitudes, comportamentos, predisposições e interações. Os autores ressaltam a significativa participação da cultura na expressão da sexualidade uma vez que ela envolve papéis sociais e é permeada por crenças, mitos, valores e atitudes. Assim, a sexualidade é uma experiência individual, porém inserida em específicos contextos sociais e coletivos (Araújo et al., 2013; Vieira, Miranda, \& Coutinho, 2012).

Debert e Brigeiro (2012) destacam que, a despeito do sentido atribuído comumente a sexualidade no envelhecimento (inexistência, ausência de libido e 
de relações sexuais), pesquisas recentes concordam que a sexualidade não cessa com o passar dos anos e ao longo da vida. Nesse sentido, a sexualidade não se limitaria às funções reprodutivas, pois é impulsionada por fatores como a realização do desejo que não se esgota com a idade e a constante busca por prazer (Santos, 2011).

O conceito de gênero é importante para a compreensão da sexualidade. Segundo Butler (2012), gênero é fruto de construções socioculturais que correspondem a interesses políticos, econômicos e sociais que visam adequar os sujeitos às regras que estipulam comportamentos e características considerados tipicamente femininos e masculinos. Assim, para a autora, o gênero é um mecanismo que estrutura as relações sociais e permite que as noções de masculino e feminino sejam naturalizadas por meio das repetições de gestos, movimentos, signos, normas e estilos culturais.

Butler (2012) considera tanto o sexo como o gênero construções discursivas e culturais que, nas sociedades contemporâneas, impõem uma ordem compulsória e heterossexual que pressiona a coesão entre sexo biológico (anatomia), gênero (papel) e práticas/desejos (orientação sexual). Evidencia-se assim que os códigos sociais e os valores tradicionais atribuídos aos gêneros masculino e feminino (propagados pelos discursos hegemônicos e processos educativos) extrapolam as diferenças biológicas, interferem no modo como cada indivíduo vivencia sua sexualidade, seus corpos e desejos, e geram exclusões dos que não se adéquam a essas normas. Assim, tais tradicionalismos contribuem para a construção de relações de poder diferentes e assimétricas entre os gêneros (Butler, 2012; Silva, \& Santiado, 2014).

Desse modo, pode-se dizer que provavelmente os atuais idosos viveram em um contexto em que homens e mulheres possuíam papéis denominados tradicionais de gênero, visto que os homens eram responsáveis pelo trabalho, por proverem e protegerem suas famílias e deveriam atuar na esfera pública. Já as mulheres estavam inseridas na esfera privada devendo exercer as atividades domésticas e os cuidados da família. Ressalta-se que neste contexto a sexualidade feminina foi atrelada à reprodução biológica e à maternidade e a sexualidade masculina aos valores da esfera pública (Moraes et al., 2011). Esses predicados podem influenciar a vivência da sexualidade na menopausa.

O climatério representa uma fase do ciclo de vida das mulheres marcada pela transição biológica entre o período reprodutivo e o não reprodutivo. $\mathrm{O}$ organismo passa por adaptações a um novo meio hormonal e emocional e, desse modo, as mulheres vivenciam alterações hormonais e metabólicas que podem ser acompanhadas por mudanças somáticas, psíquicas e relacionais (Nappi, \& Lachowsky, 2009; Valença, Nascimento Filho, \& Germano, 2010).

A menopausa é um episódio pontual durante o climatério e se caracteriza pela finalização permanente das menstruações após um período de 12 meses consecutivos. Ela pode ocorrer de forma espontânea (em torno de 45 a 50 anos de idade) ou em decorrência de intervenções médicas. Os sintomas mais comuns são: ondas de calor, sudorese, secura vaginal, dificuldades de excitação e orgasmo e alterações no funcionamento sexual. Contudo, a maneira como cada mulher vivencia este período não se limita às influências biológicas, pois um complexo conjunto de fatores contextuais e pessoais (hábitos de vida, história pessoal etc.) também está associado a essa experiência (Brasil, 2008; Nappi, \& Lachowsky, 2009). Por isso é importante compreender as influências psicossociais, emocionais, culturais e situacionais que acompanham as mulheres após a menopausa e podem influenciar sua sexualidade, uma vez que a autoimagem, o papel social feminino e as relações sociais ganham novos contornos e significações nesta etapa da vida (Valença et al., 2010).

Desse modo, Biasus, Demantova e Camargo (2011) destacam a importância de questionar a perspectiva negativa e os mitos acerca da menopausa, tais como a assexualidade da mulher e a ausência de erotismo e de desejo sexual (libido). Nesse sentido, compreender os papéis de gêneros faz-se relevante, pois historicamente é possível identificar a maior valorização da mulher no período reprodutivo do que nas fases consideradas não reprodutivas, o que demarca a associação da sexualidade feminina à reprodução e fertilidade. Tais estereotipias podem interferir negativamente na autopercepção da mulher e na sua sexualidade (Valença et al., 2010).

Além disso, a educação e acesso às informações sobre sexualidade e processos biológicos e emocionais da menopausa são importantes para a compreensão e adaptação a essas mudanças. Pesquisas epidemiológicas evidenciam que a inserção das mulheres em contextos educacionais e de orientação qualificada possibilita que vivenciem melhor a menopausa e suas consequências, possibilitando melhor qualidade de vida (Oliveira, Jesus, \& Merighi, 2008; Valença et al., 2010). 
Fleury e Abdo (2015) salientam que a população idosa feminina apresenta significativos comprometimentos em relação à sexualidade quando comparada aos homens, tais como a diminuição na qualidade de vida sexual e interesse sexual reduzido ao longo dos anos, especialmente por parte de mulheres solteiras ou viúvas. Os autores pontuam que esses aspectos podem contribuir para o abandono da própria vida sexual. Desse modo, evidencia-se a importância da realização de uma revisão integrativa da literatura nacional e internacional sobre os aspectos envolvidos na sexualidade de idosas que já passaram pela menopausa, a fim de avaliar as possíveis repercussões da mesma para a vida sexual, para a qualidade de vida e para seus relacionamentos íntimos.

Entende-se ainda que essa revisão integrativa possa contribuir para um aprofundamento e sistematização do conhecimento do tema, bem como para uma análise crítica do que tem sido produzido em relação à sexualidade de mulheres idosas após a menopausa. Assim, o objetivo do presente estudo foi apresentar uma revisão integrativa da literatura científica nacional e internacional sobre as possíveis repercussões da menopausa para a sexualidade de idosas.

\section{Método}

\section{Tipo de estudo}

Trata-se de uma revisão integrativa da literatura científica. Este tipo de revisão pretende apresentar uma síntese de diversas pesquisas publicadas possibilitando expressar conclusões gerais a respeito de um tema e promover discussões críticas sobre os métodos, objetivos e resultados dessas pesquisas (Broome, 2000). A revisão integrativa é um instrumento estabelecido por meio da prática baseada em evidências (PBE) e é composta pelas seguintes etapas (Mendes, Silveira, \& Galvão, 2008): (1) identificação do tema e seleção da questão norteadora da pesquisa, (2) estabelecimento de critérios de inclusão e exclusão da amostra, (3) busca nas bases de dados e busca de textos na íntegra, (4) definição das informações a serem utilizadas dos estudos selecionados, (5) avaliação dos estudos incluídos e categorização das informações encontradas, (6) interpretação dos resultados e (7) apresentação da revisão do conhecimento.

\section{Bases indexadoras, unitermos e critérios de inclusão/exclusão}

Realizou-se uma busca sistemática nas bases de evidências da Literatura Latino-Americana e do Caribe em Ciências da Saúde (Lilacs), na Scientific Electronic Library Online (SciELO), nos Periódicos Eletrônicos de Psicologia (PePSIC) e na American Psychological Association (PsycINFO) a fim de garantir a abrangência da revisão. A questão norteadora desta pesquisa foi: Quais as possíveis repercussões da menopausa para a sexualidade de mulheres idosas?, sendo as mulheres idosas a população alvo (P), a menopausa a intervenção avaliada (I) e as possíveis repercussões para a sexualidade o resultado esperado (O) (Santos, Pimenta,\& Nobre, 2007).

Foram utilizados descritores e palavras-chave em português e seus correspondentes em inglês e espanhol: menopausa (menopause e menopausia), sexualidade (sexuality e sexualidad), sexualidade feminina (femal e sexuality e sexualidad feminina), atividade sexual (sexual activity e actividad sexual), climatério (climateric e climatério), envelhecimento (aging e envejecimiento), mulher (women e mujer) e idoso (elder, aged e anciano). Estes descritores e seus correspondentes em inglês e espanhol foram combinados pelo operador booleano and das seguintes formas: climatério OR climateric OR climatério AND atividade sexual OR sexual activity OR actividad sexual (exemplo de busca realizada com todas as combinações); climatério e envelhecimento; menopausa e sexualidade; menopausa e sexualidade feminina; menopausa e atividade sexual; menopausa e mulher; menopausa e idoso; menopausa e envelhecimento; menopausa e sexualidade e envelhecimento; sexualidade e climatério; sexualidade e mulher; sexualidade e idoso; sexualidade e envelhecimento; sexualidade feminina e climatério; sexualidade feminina e envelhecimento; sexualidade feminina e idoso. Todas estas 16 combinações foram empregadas nas buscas realizadas nas bases Lilacs, SciELO e PepSIC, e na base PsycINFO 11 destas combinações foram utilizadas.

A busca nas bases foi realizada durante o mês de abril de 2016, sendo que a coleta em cada base foi conduzida pelos pesquisadores e um juiz independente a fim de avaliar a compatibilidade dos dados encontrados. Os critérios de inclusão foram: (a) artigos disponibilizados na íntegra, (b) publicados entre janeiro de 2006 até março de 2016, (c) redigidos nos idiomas português, inglês e espanhol, (d) que aborda- 
vam mulheres com idade a partir de 60 anos na amostra, e (e) com temática compatível que permitisse responder a questão norteadora e o objetivo da revisão.

Foram excluídos todos os artigos que (a) estavam fora do período de publicação elegido, (b) em idiomas diferentes do português, inglês ou espanhol, (c) publicações do tipo revisão de literatura, estudos teóricos e atualizações, (d) outros tipos de publicações que não artigos (livros, capítulos, monografias, dissertações, teses, resenhas, cartas e notícias), (e) artigos que abordavam apenas mulheres com idade inferior a 60 anos, (f) artigos repetidos e, por fim, (g) artigos que apresentavam os descritores e palavras-chave alvos, mas não respondiam a questão norteadora e o objetivo da revisão.

\section{Procedimentos}

Inicialmente realizou-se a leitura minuciosa dos títulos das publicações localizadas. Foram excluídos os artigos que não correspondiam aos critérios de inclusão e os motivos das exclusões foram listados. Em seguida, procedeu-se a leitura dos resumos dos artigos restantes e foram realizados os mesmos procedimentos de exclusão aplicados aos títulos. Por fim, os artigos selecionados foram recuperados e lidos na íntegra. Desse modo, após avaliação destas publicações apenas os artigos diretamente relacionados ao tema e a questão norteadora desta revisão foram selecionados para compor o corpus final de análise.

As principais características (título, autores, ano de publicação, periódico, base de dados, objetivo, tipo de estudo, amostra, instrumentos, principais resultados e conclusões) dos artigos selecionados para a revisão foram sistematizadas em uma planilha visando auxiliar a constituição das categorias de análise. Assim, três categorias temáticas foram organizadas mediante a semelhança de conteúdos (Turato, 2008): Percepções das mulheres idosas sobre as repercussões da menopausa; Impactos da menopausa para a prática sexual; Função sexual após a menopausa e Fatores associados às disfunções.

\section{Resultados e Discussão}

\section{Descrição quantitativa}

Nesta etapa são apresentados os principais aspectos que caracterizam os procedimentos de composição da amostra bem como o perfil da amostra final de artigos. Assim, a Figura explicita o fluxograma do processo de revisão, compreendendo o montante das produções localizadas na busca bem como os momentos e motivos de exclusão de algumas destas produções.

Assim, a partir dos artigos encontrados $(\mathrm{n}=4094)$, constata-se que a maioria foi excluída com base nos títulos ( $\mathrm{n}=3823$, ou $93,38 \%$ ), e dentre os excluídos muitos ( $\mathrm{n}=3273$, ou $85,61 \%$ ) o foram devido ao distanciamento do tema desta revisão e da questão norteadora da mesma, e tratavam de temáticas tais como: mulheres com idade inferior a 60 anos ou adolescentes; associação da menopausa com câncer de mama, incontinência urinária, osteoporose, depressão, obesidade; homens e andropausa; questões de gênero e orientação sexual; doenças sexualmente transmissíveis e outras temáticas relacionadas à sexualidade (contracepção, gestação, aborto e amamentação). Todavia, destaca-se o contingente significativo de pesquisas que investigavam os aspectos patológicos envolvidos no processo da menopausa.

Outros motivos de exclusões com base no título foram: as revisões de literatura $(\mathrm{n}=110$, ou $2,87 \%$ ), as produções de outros tipos que não artigos (dissertações, resumos de congressos e editoriais) ( $\mathrm{n}=8$, ou $0,2 \%$ ), os títulos repetidos entre as bases ( $n=396$, ou $10,35 \%$ ), as publicações em idiomas diferentes dos estipulados ( $n=12$, ou $0,31 \%$ ) e outros por não estarem disponíveis gratuitamente $(\mathrm{n}=24$, ou $0,62 \%)$.

Após as exclusões com base nos títulos passou-se à leitura dos resumos dos 271 artigos restantes, dos quais $171(63,09 \%)$ foram excluídos. As razões para essas exclusões foram: não tratar especificamente da pergunta norteadora desta pesquisa e abordar diagnósticos de doenças relacionadas à menopausa ( $n=33$, ou 19,29\%) e efeitos das medicações e procedimentos ( $\mathrm{n}=30$, ou 17,54\%); também foram excluídos os que abordavam apenas mulheres com idade inferior a 60 anos ( $\mathrm{n}=34$, ou $19,88 \%)$, os que referiam às questões de gênero ( $\mathrm{n}=7$, ou 4,09\%) sem investigar as repercussões da menopausa ( $\mathrm{n}=32$, ou $18,71 \%)$; as revisões de literatura $(\mathrm{n}=18$, ou $10,52 \%)$ e outros tipos de estudo como editoriais ( $n=1,0,58 \%$ ), validações de instrumentos ( $\mathrm{n}=6$, ou $3,50 \%)$, bem como artigos não encontrados ( $\mathrm{n}=3$, ou $1,75 \%)$ ou não disponibilizados gratuitamente ( $\mathrm{n}=7$, ou $4,09 \%)$.

Desse modo, após a exclusão pela leitura dos resumos 100 artigos foram selecionados para leitura na íntegra e desses 36 compuseram o corpus final desta revisão. Os demais 64 artigos $(\mathrm{n}=64$, ou 64\%) foram excluídos por: abordarem a sexualidade na 


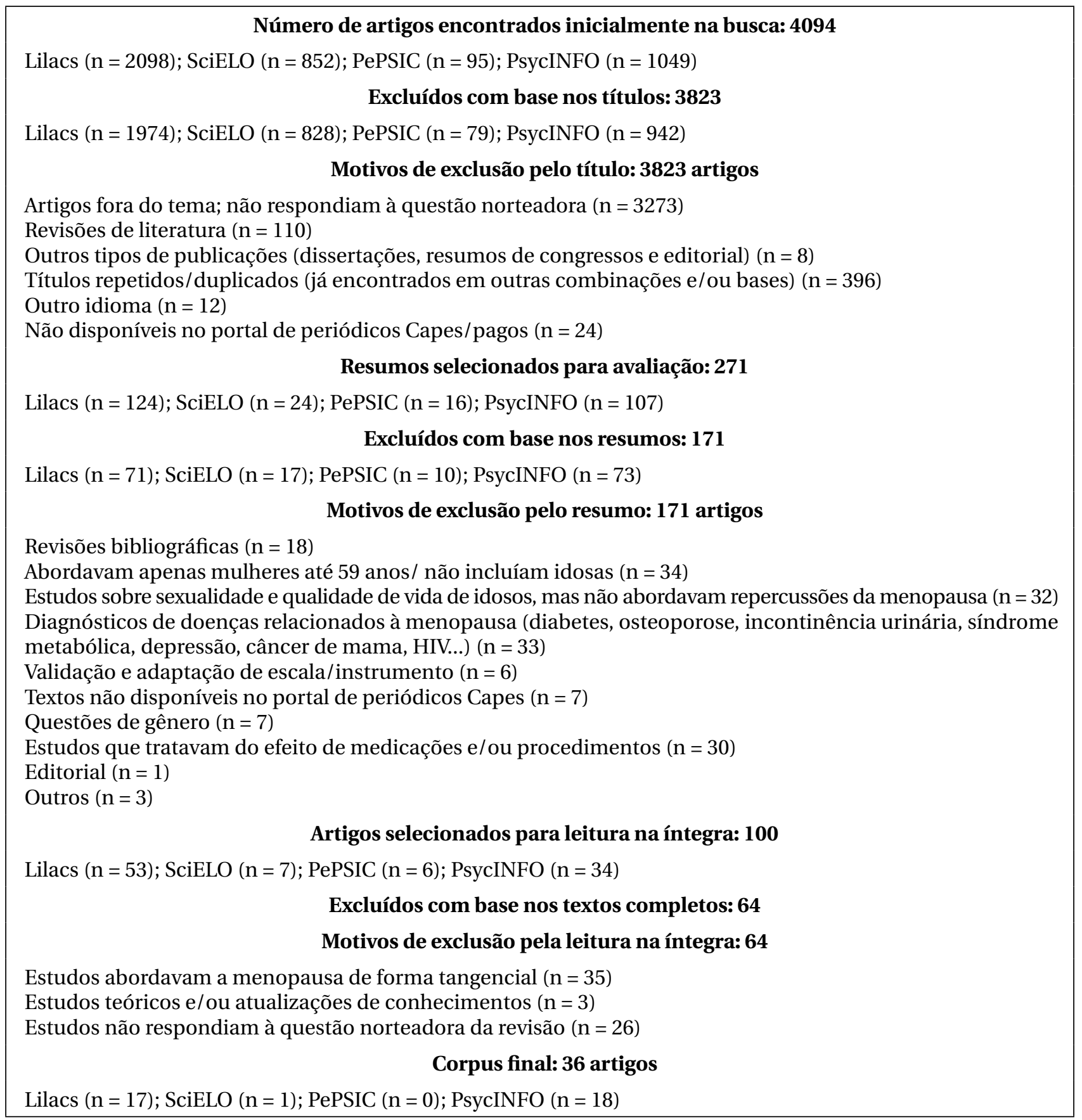

Figura.

Fluxograma do processo de revisão.

menopausa de forma tangencial ( $\mathrm{n}=35$, ou $54,68 \%$ ), por serem estudos teóricos e/ou atualizações de conhecimentos (estudos teóricos) ( $\mathrm{n}=3$, ou 4,68\%), e/ou por não responderem à questão norteadora da pesquisa ( $n=26$, ou $40,62 \%$ ). Essas exclusões destacam novamente o número expressivo de estudos voltados para mulheres com idade inferior a 60 anos e para as patologias e medicalização da menopausa.
Tais dados indicam uma tendência em associar a sexualidade feminina aos papéis tradicionais de gênero, aos aspectos biológicos e à reprodução biológica direcionada as mulheres jovens e férteis. Nesse sentido, as patologias e as possibilidades medicamentosas são enfatizadas em grande parte dos estudos excluídos, os quais exploram as temáticas da menopausa e do envelhecimento a partir dessas perspectivas. 
Sobre o perfil da amostra final, composta por 36 artigos, 17 artigos foram recuperados da base de dados Lilacs, 18 da PsycINFO e 1 da SciELO, sendo 20 artigos em idioma inglês (55,5\%), 14 em português $(38,8 \%)$ e 2 em espanhol $(5,55 \%)$. Nota-se a expressividade da produção internacional acerca da temática desta revisão, principalmente no idioma inglês. Tal fato possivelmente sinaliza a maior concentração de interesses e investimentos voltados para a produção científica neste tema, aspectos que impactam diretamente a publicação de pesquisas. Todavia, as publicações em português representam $38,88 \%$ da amostra, demonstrando uma participação importante voltada para os estudos sobre menopausa e sexualidade.

Em relação ao ano de publicação dos artigos constatou-se: um artigo em 2006, cinco artigos em 2007, sete artigos em 2008, cinco artigos em 2009, dois artigos em 2010, dois artigos em 2011, três artigos em 2012, quatro artigos em 2013, três artigos em 2014 e quatro artigos em 2015, havendo prevalência de publicações dentre 2007 a 2009 com 17 produções (47,22\%). Apenas no ano de 2016 não foram encontradas publicações pertinentes ao objetivo desta revisão, possivelmente devido ao fato de que, quando a busca nas bases foi realizada, aquele ano ainda estava em curso. O periódico The Journal of Sexual Medicine foi responsável pela publicação de 10 artigos (27,77\%) da amostra, destacando a expressiva publicação de informações científicas geradas a partir de investigações experimentais e clínicas (escopo daquele periódico).

Por sua vez, a maior parte dos artigos que compuseram a amostra final é representada por estudos de caráter quantitativo ( $\mathrm{n}=23$, ou $63,88 \%$ ), com número significativo de pesquisas que utilizaram delineamentos descritivos, corte transversal e tamanho amostral elevado. Isso pode ser compreendido como uma tentativa de realizar tratamento estatístico dos resultados e possibilitar generalizações e o estabelecimento de um perfil para as repercussões da sexualidade após a menopausa. Além disso, os estudos qualitativos selecionados $(n=12$, ou $33,33 \%)$ foram realizados com amostras menores e estavam direcionados para a compreensão dos sentidos e posicionamentos das idosas acerca das repercussões da menopausa para a sexualidade.

Ressalta-se que as idades das participantes e a conceituação de climatério e menopausa, bem como o período correspondente a cada fase, apresentaram distinções entre as pesquisas que compuseram a amostra final e respondiam às concepções teóricas e metodológicas de seus autores. Desse modo, cada pesquisa apresentou participantes com diferentes faixas etárias e as idades variaram de 18 a 90 anos. Foram consideradas todas as perspectivas dos autores, independentemente da sua conceituação, a fim de permitir a integração dos conhecimentos. Ademais, todos os artigos abordaram em suas amostras mulheres com idade superior a 60 anos e os resultados pertinentes a essas participantes foram enfatizados na análise desta revisão.

Dos 36 artigos apenas seis (16,66\%) trataram exclusivamente de mulheres idosas no período pós-menopausa. Dos demais, um artigo $(2,77 \%)$ abordou homens e mulheres idosos, um artigo (2,77\%) realizou a coleta de dados com mulheres e médicos ginecologistas e 28 (77,77\%) enfatizaram mulheres de diferentes faixas etárias além das idosas, com foco para a menopausa. Constata-se assim, um número reduzido de produções voltadas apenas para as mulheres idosas, suas percepções e as possíveis repercussões ao longo prazo da menopausa para a sua sexualidade.

\section{Descrição qualitativa}

Nesta etapa os artigos selecionados foram agrupados mediante a semelhança dos seus conteúdos, isto é, eles foram organizados nas seguintes categorias temáticas: percepções das mulheres idosas sobre as repercussões da menopausa; impactos da menopausa para a prática sexual; função sexual após a menopausa e fatores associados às disfunções.

\section{Percepções das mulheres idosas sobre as repercussões da menopausa}

A partir dos 36 artigos selecionados constatou-se que 12 artigos se aproximaram por terem como intuito compreender os significados, conhecimentos, percepções e vivências atribuídas pelas mulheres sobre a menopausa e suas repercussões, principalmente para a sexualidade (Almeida, Luz, \& Monteiro, 2007; Araújo et al., 2013; Coelho, Daher, Santana, \& Santo, 2010; Costa, \& Gualda, 2008; Feltrin, \& Velho, 2014; Frugoli \& Magalhães-Júnior, 2011; Hinchliff, \& Gott, 2008; Kisa, Zeynelog, \& Ozdemir, 2012; Trench \& Rosa, 2008; Valadares et al., 2013; Wood, Mansfield, \& Koch, 2007; Zampieri et al., 2009).

Tais estudos possibilitaram a expressão destas mulheres e a valorização de seus discursos, viabilizando resultados contextualizados e coerentes com a 
realidade das mesmas. Desse modo, o climatério e a menopausa foram abordados como períodos naturais e esperados para o processo de envelhecimento feminino, porém intimamente conectados aos aspectos culturais, às exigências e expectativas sociais, a vivência da corporeidade e aos relacionamentos afetivos estabelecidos ao longo da vida, além dos mitos, tabus, crenças e conhecimentos apresentados por cada mulher (Hinchliff, \& Gott, 2008; Valadares et al., 2013).

Nesse sentido, foi recorrente entre estes estudos que as repercussões da menopausa devem ser compreendidas considerando o contexto de vida dessas mulheres, além dos fatores biológicos que, segundo elas, não devem ser descartados. Trata-se, portanto, de um processo singular, mas influenciado pela cultura e sociedade. No entanto, as possíveis mudanças e sintomas corporais (secura vaginal, dor e desconforto nas relações sexuais, fogachos, alterações da libido, sudorese, insônia, dor nas pernas, sangramento vaginal, aumento de peso, depressão, perda da capacidade reprodutiva e alterações na vivência da sexualidade) foram destacados em todos os estudos, visto que sinalizam biologicamente o processo da menopausa. Nota-se assim, forte associação da menopausa às patologias e suas influências para a sexualidade ao longo do envelhecimento e, consequentemente, a necessidade da medicalização para o enfrentamento dos sintomas (Almeida et al., 2007; Feltrin, \&Velho, 2014; Trench, \& Rosa, 2008; Wood et al., 2007).

Ressalta-se que as alterações hormonais e os discursos biomédicos (amplamente difundidos aos pacientes) aliados à falta de informações e esclarecimentos sobre a menopausa foram fatores destacados pelas participantes daquelas pesquisas como influências significativas para o entendimento da menopausa como fenômeno essencialmente biológico, marcado por doenças, alterações dos corpos, dos desejos das mulheres e com implicações negativas sobre a sexualidade (Costa, \& Gualda, 2008; Feltrin, \& Velho, 2014; Frugoli, \& Magalhães-Júnior, 2011).

Evidenciou-se em grande parte dos artigos a preocupação das mulheres acerca das repercussões do climatério e da menopausa para o envelhecimento. Além disso, as mulheres destacaram a menopausa como uma experiência negativa e associada à diminuição do desejo e do prazer sexual, às mudanças estéticas, à perda da atração e da sensualidade e principalmente a modificação do papel social feminino (Araújo et al., 2013; Hinchliff, \& Gott, 2008; Kisa et al., 2012; Trench, \& Rosa, 2008; Zampieri et al., 2009).
Apesar dessas constatações das participantes, elas também abordaram a menopausa como um fenômeno complexo e ambíguo em suas vidas - isso foi referido principalmente pelas idosas que passaram por diversas experiências e adaptações ao longo da vida. Tal processo envolveu perdas, ganhos e ressignificações e, portanto, a menopausa não deve ser limitada aos fatores biológicos e patológicos naturalizados pelos discursos biomédicos ainda presentes na prática clínica. Assim, a partir das percepções e relatos das mulheres três temáticas se destacaram como elementos significativos para a compreensão sobre a vida sexual após a menopausa: em primeiro lugar a influência das informações insuficientes sobre a menopausa, sobre o próprio corpo e sobre sexualidade; em segundo lugar o papel das expectativas sociais e das frustrações pessoais diante dos papéis tradicionais de gênero; em terceiro lugar a importância do apoio social para a vivência desta experiência ao longo da vida (Araújo et al., 2013; Coelho et al., 2010; Feltrin, \& Velho, 2014; Frugoli, \& Magalhães-Júnior, 2011; Wood et al., 2007).

Considera-se que o tema sexualidade envolve diversos mitos e tabus e que a educação de grande parte das atuais mulheres idosas foi marcada de forma expressiva pela ausência de diálogo sobre esses assuntos, pela maior rigidez, pelos tradicionalismos e preconceitos de gênero, além das dificuldades de acesso às informações e esclarecimentos sobre assuntos como a menopausa e a corporeidade. Desse modo, os discursos das mulheres desses estudos revelaram dúvidas, incertezas e desinformações durante e após a menopausa. Tais aspectos possivelmente contribuíram para a construção de perspectivas distorcidas sobre a sexualidade após a menopausa (Araújo et al., 2013; Costa, \& Gualda, 2008; Feltrin, \& Velho, 2014; Frugoli, \& Magalhães-Júnior, 2011; Zampieri et al., 2009). Kisa et al. (2012) evidenciaram uma significativa relação entre a percepção da menopausa e o nível educacional das mulheres, visto que as participantes com maior nível educacional e acesso às informações demonstraram atitudes mais positivas em relação à menopausa e a própria sexualidade.

Outro aspecto importante discutido pelos e a partir dos estudos que compõem essa categoria diz respeito aos papéis de gênero e às expectativas sociais propagadas em relação às funções, posicionamentos e a própria sexualidade feminina, visto que a capacidade de reprodução biológica, as menstruações e a 
jovialidade historicamente representam para a sociedade ocidental fertilidade, associada à sexualidade das mulheres jovens. Nesse sentido, a menopausa foi significada por grande parte das participantes como um período de declínio da atratividade e do exercício sexual e de um distanciamento dos padrões estéticos e das funções esperadas como naturais para as mulheres (nominalmente, a maternidade), além da maior importância da satisfação do desejo sexual masculino em detrimento das próprias vontades. Neste contexto, insatisfações com os relacionamentos afetivos e sentimentos de inadequação demonstraram-se relevantes para a compreensão da sexualidade das mulheres idosas e das repercussões da menopausa em suas vidas (Araújo et al., 2013; Coelho et al., 2010; Feltrin, \&Velho, 2014; Valadares et al., 2013; Zampieri et al., 2009).

Contudo, essa premissa (apesar de frequente na maioria dos relatos) não deve ser generalizada, pois também foram encontrados resultados dissonantes, tais como os relatos de algumas mulheres que afirmaram que após a menopausa foram impulsionadas a buscar maior autonomia, mudanças e novas conquistas, inclusive no que se refere à sexualidade. Elas mencionaram que redescobriram seus próprios corpos, possibilidades de prazer, satisfações dos seus desejos e autocuidado, reconheceram-se mais vaidosas e livres dos ciclos menstruais e da possibilidade de gravidez. Constata-se assim que a menopausa também pode gerar repercussões positivas para a sexualidade das mulheres (Almeida et al., 2007; Kisa et al., 2012; Zampieri et al., 2009). Neste contexto o apoio dos parceiros, familiares, grupos de convivência e de aprendizado e o contato com profissionais de saúde e da educação revelaram-se fundamentais, pois permitem o fortalecimento e a valorização destas mulheres por meio da compreensão, da escuta livre de preconceitos, do respeito e do suporte frente as suas dificuldades e dúvidas (Coelho et al., 2010; Wood et al., 2007).

\section{Impactos da menopausa para a prática sexual}

Após avaliação da amostra constatou-se que 12 artigos buscaram de modo semelhante avaliar as repercussões da menopausa para as práticas sexuais principalmente em relação aos seus aspectos físicos e biológicos (Alves et al., 2015; Burri, Hilpert, \& Spector, 2015; Correa et al., 2012; Davison, Bell, LaChina, Holden, \& Davis, 2009; Fonnegra, \& Bojacá, 2009; Gradim, Sousa, \& Lobo, 2007; Kokcu et al., 2015; McCall,
\& Meston, 2007; McCall-Hosenfeld et al., 2008; Trindade, \& Ferreira, 2008; Woloski-Wruble, Oliel, Leefsma, \& Hochner-Celnikier, 2010; Yucel, \& Eroglu, 2013). Para tanto foi destacada a continuidade da sexualidade em idades avançadas e as possibilidades de manutenção das práticas sexuais ao longo de toda vida, porém considerando as particularidades e as transformações decorrentes do processo de envelhecimento e da menopausa (Gradim et al., 2007; Woloski-Wruble et al., 2010; Yucel, \& Eroglu, 2013).

A maioria destes estudos (dez) utilizou a abordagem quantitativa e comparações entre grupos a fim de mensurar e diferir variáveis. Assim, foram abordados diversos grupos, além das amostras somente de idosas, tais como mulheres na pré e na pós-menopausa (Burri et al., 2015; McCall, \& Meston, 2007), mulheres que vivenciaram a menopausa de maneira natural ou cirúrgica (Correa et al., 2012; Kokcu et al., 2015) e grupos de diferentes faixas etárias (Davison et al., 2009).

Nesse sentido, observou-se a tentativa de estabelecer parâmetros e comparações para determinar as repercussões e os níveis de modificações da menopausa sobre o orgasmo, lubrificação vaginal, excitação, frequência de atividade sexual, desejo, dor e desempenho sexual dessas mulheres ao longo dos anos, bem como os níveis de satisfação e insatisfação sexual (Alves et al., 2015; Kokcu et al., 2015; McCall-Hosenfeld et al., 2008). Mesmo considerando a importância da mensuração de tais aspectos e suas influências para a sexualidade foram evidenciadas limitações nos resultados desses estudos - pois ao enfatizarem a sintomatologia, os aspectos psicossociais, culturais e emocionais da sexualidade (intimamente relacionados aos fatores fisiológicos) não foram contemplados pelos instrumentos e escalas utilizados (Burri et al., 2015).

Ressalta-se que todos os estudos que compuseram essa categoria tiveram suas hipóteses iniciais calcadas nas alterações hormonais e fisiológicas consideradas típicas do envelhecimento feminino, especialmente o declínio da produção de estrogênio (que pode ocorrer durante a menopausa e causar em longo prazo prejuízos para a resposta sexual feminina), a diminuição da frequência sexual e do desejo sexual e o aparecimento de sintomas desagradáveis como dores e ressecamento vaginal. Portanto, o envelhecimento foi considerado um fator para a ocorrência de tais alterações (Gradim et al., 2007; Trindade, \& Ferreira, 2008). Desse modo, cinco dos 12 estudos confirmaram tal hipótese, evidenciando em suas 
amostras: declínio significativo na frequência das relações sexuais e do prazer, alta ocorrência de disfunções sexuais e dor durante o ato sexual, além de diminuição da lubrificação vaginal e menor intensidade de orgasmos com o avanço da idade das participantes. Tais resultados corresponderam a grandes amostras e geraram dados estatísticos consistentes (Fonnegra, \& Bojacá, 2009; Yucel, \& Eroglu, 2013). Por exemplo, no estudo de Yucel e Eroglu (2013) com 309 mulheres turcas a frequência de relações sexuais após a menopausa diminuiu para $80 \%$ das mulheres e o prazer foi prejudicado para $62 \%$ da amostra.

Observa-se ainda a associação entre a satisfação sexual e o bem-estar, visto que as mulheres que sofreram essas mudanças e se declaram sexualmente insatisfeitas também apresentaram bem-estar psicológico e vitalidade comprometidos (Davison et al., 2009). Já em relação à ocorrência da menopausa de forma natural ou cirúrgica houve divergências entre os estudos: Kokcu et al. (2015) não constataram resultados estatisticamente discrepantes no desempenho sexual entre esses grupos (menopausa natural e menopausa cirúrgica), com exceção da lubrificação vaginal que foi menor para mulheres que passaram pela menopausa cirúrgica. Já Correa et al. (2012) encontraram melhores pontuações nos instrumentos para a resposta sexual de mulheres que vivenciaram a menopausa natural, principalmente em relação a excitação e ao orgasmo, além de apresentarem melhores índices de bem-estar sexual e qualidade de vida quando comparadas às mulheres que realizaram cirurgicamente a retirada total ou parcial do útero (histerectomia). Diante destas divergências, acredita-se que fatores como o histórico de vida das mulheres, a cultura, os valores, a qualidade dos seus relacionamentos, o desempenho sexual ao longo da vida e a educação recebida podem contribuir para as diferenças apresentadas entre os estudos e grupos. Porém, tais aspectos não foram o foco de análise naquelas pesquisas, apesar de citados como prováveis fontes de influência.

A respeito da hipótese que perpassou os estudos cinco deles encontraram resultados que permitiram questionamentos e contraposições a ela, visto que foram evidenciadas possibilidades de mudanças positivas, maior satisfação sexual e melhor qualidade na vivência da sexualidade entre as mulheres idosas, mesmo que tenham sido elencadas manifestações leves de sintomas, alterações discretas na excita- ção, no orgasmo e no prazer sexual (Alves et al., 2015; Woloski-Wruble, et al., 2010). Por exemplo, no estudo de McCall-Hosenfeld et al. (2008) realizado com 46.526 participantes, $77 \%$ demonstraram satisfação sexual com o aumento da idade, principalmente as mulheres entre 70 e 79 anos emocionalmente saudáveis e envolvidas em um relacionamento. Além disso, manifestações mais frequentes de amor e satisfação emocional com seus parceiros foram destacadas pelas mulheres idosas como elementos positivos para o enfrentamento da pré e pós-menopausa (McCall, \& Meston, 2007).

Constatou-se ainda a partir do estudo longitudinal sobre as repercussões da menopausa desenvolvido durante quatro anos por Burri et al. (2015) que, ao longo dos anos e dos ciclos de vida, o funcionamento sexual feminino também pode apresentar estabilidade, sem alterações severas quando comparado aos outros períodos da vida. Desse modo, tanto problemas sexuais como melhorias foram evidenciadas pelas mulheres na pré e na pós-menopausa daquele estudo. Por fim, é possível considerar que as melhorias na sexualidade das mulheres ao longo dos anos podem estar associadas a diversos fatores para além das sintomatologias previstas (geralmente de caráter negativo), tais como: a maior intimidade e melhor comunicação nos relacionamentos íntimos, o desenvolvimento de estratégias para melhorar a própria vida sexual, o maior conhecimento do próprio corpo e dos desejos, a disponibilidade para vivenciar a sexualidade e a sensualidade para além dos padrões estabelecidos socialmente (Gradim et al., 2007; McCall, \& Meston, 2007; Woloski-Wruble et al., 2010).

Assim, não são poucos os estudos que revelam que as mulheres idosas apesar das repressões, dos padrões sociais que constrangem a expressão da sexualidade e dos valores tradicionais de gênero que compuseram suas vidas, atualmente estão abertas para modificar, dialogar, refletir e redescobrir a sexualidade a fim de alcançar maior liberdade e satisfação (Fonnegra, \& Bojacá, 2009; Gradim et al., 2007).

\section{Função sexual após a menopausa e fatores associados às disfunções}

Durante a menopausa ocorrem alterações hormonais importantes, principalmente a diminuição dos níveis de estrogênio. Essas mudanças podem resultar em significativas disfunções sexuais e repercussões para o funcionamento sexual de muitas mulheres, 
afetando a sexualidade das mesmas. Desse modo, essa categoria está composta por 12 artigos que em comum possuem o intuito de avaliar a função sexual das mulheres após a menopausa (Cabral et al., 2012; Cavalcanti, Farias, Ithamar, Silva, \& Lemos, 2014; Dennerstein, Koochaki, Barton, \& Graziottin, 2006; Erenel, Golbasi, Kavlak, \& Dilbaz, 2015; Graziottin, 2007; Graziottin, Koochaki, Rodenberg, \& Dennerstein, 2009; Hayes et al., 2008; Lonnèe-Hoffmann, Dennerstein, Lehert, \& Szoeke, 2014; Polizer, \& Alves, 2008; Silva, Lima, \& Moraes, 2013; Thompson et al., 2011; Valadares et al., 2008).

Alguns deles pretendem compreender os principais fatores associados às disfunções sexuais em diferentes situações envolvendo mulheres que: ou passaram pela menopausa natural ou cirúrgica em diferentes países (Dennerstein et al., 2006; Graziottin, 2007; Graziottin et al., 2009); ou possuem problemas de saúde (síndrome metabólica, incontinência urinária e hipertensão arterial sistêmica, por exemplo) (Cavalcanti et al., 2014; Silva et al., 2013); ou mulheres de diferentes faixas etárias com ou sem risco de disfunção sexual (Cabral et al., 2012; Valadares et al., 2008).

Nota-se entre a maioria dos estudos o reconhecimento das possíveis influências destas disfunções sexuais para a vida das mulheres e a influência dos sintomas da menopausa para a acentuação destas disfunções, cuja finalidade é respaldar a prática clínica e proporcionar maiores informações e recursos interventivos adequados para as mulheres acometidas por tais dificuldades (Lonnèe-Hoffmann et al., 2014). Assim, os níveis de satisfação em relação à sexualidade também foram avaliados, visto que esse aspecto está interligado à função sexual (Polizer, \& Alves, 2008; Thompson et al., 2011). Nesse sentido, as principais disfunções investigadas foram: Hypoactive Sexual Desire Disorder (HSDD) ou frigidez (caracterizada como o diminuto desejo sexual que causa sofrimento e preocupações a mulher) e as disfunções associadas às alterações severas que acometem o desejo sexual, a excitação, a lubrificação, o orgasmo, a satisfação e outros que causam dores durante o ato sexual.

Avaliações de mulheres com ou sem risco de disfunções sexuais permitiram reflexões. Foi possível observar os impactos causados pela redução do desejo sexual e/ou outras disfunções sexuais quando da menopausa, pois as mulheres que apresentavam estas características possuíam maiores chances de ter a vida sexual comprometida quando comparadas com mulheres com níveis considerados socialmente adequados de desejo, tais como: menor frequência de atividade sexual, sentimentos de insatisfação com a própria sexualidade e com os relacionamentos íntimos, frequentes estados emocionais e psicológicos negativos, além de problemas sexuais e ressecamento vaginal (Cabral et al., 2012; Dennerstein et al., 2006; Graziottin et al., 2009). Porém, constatou-se que não há consenso entre os estudos sobre a existência de uma relação causal entre menopausa e o surgimento das disfunções sexuais, pois estes estudos revelam que os sintomas da menopausa podem ser vivenciados de maneiras e intensidades distintas e há um conjunto complexo de fatores que compõem a etiologia destas disfunções (Cavalcanti et al., 2014; Lonnèe-Hoffmann et al., 2014).

Alguns estudos demonstraram que comorbidades e sintomas não decorrentes da menopausa podem igualmente contribuir para o desenvolvimento das disfunções sexuais, pois afetam a qualidade de vida das mulheres em diferentes faixas etárias. Por exemplo, os estudos de Cabral et al. (2012), Silva et al. (2013) e Valadares et al. (2008) destacaram que diabetes, hipertensão arterial, depressão, ansiedade, doenças metabólicas e cardiovasculares estão associadas ao surgimento de disfunções sexuais. Ademais, alterações nas funções sexuais podem representar um alerta sobre tais doenças. Por exemplo, Silva et al. (2013) demonstraram que a glicemia interfere no desejo sexual, excitação, lubrificação, orgasmo e satisfação das mulheres, e Cavalcanti et al. (2014) evidenciaram a associação positiva entre incontinência urinária e osteoporose às disfunções sexuais, porém o mesmo não foi constatado em relação a hipertensão arterial, depressão e diabetes. Além disso, a menopausa cirúrgica foi identificada como uma circunstância favorável para as disfunções sexuais visto que mulheres submetidas a ela possuem aumento nas probabilidades de ter HSDD, baixo desejo sexual e angústias decorrentes disto (Dennerstein et al., 2006; Graziottin, 2007; Graziottin et al., 2009).

O nível de escolaridade, a qualidade do relacionamento com o parceiro(a) e as atitudes em relação à menopausa também foram fatores recorrentes nos estudos, demonstrando a importância do contexto e do histórico de vida das mulheres quando se trata de questões sexuais. Erenel et al. (2015), Hayes et al. (2008) e Valadares et al. (2008) evidenciaram a impor- 
tância do ensino e do acesso a informações corretas para a redução dos riscos das disfunções sexuais. Nesses estudos mulheres com níveis maiores de escolaridade tiveram menores índices de disfunções sexuais, além de atitudes mais positivas sobre a menopausa. Em contrapartida, Cavalcanti et al. (2014) não encontraram tal associação.

Sobre a qualidade dos relacionamentos afetivos foram observados aspectos importantes para a compreensão das disfunções sexuais: boa comunicação com o parceiro(a), abertura para dialogar sobre suas necessidades e satisfação com o relacionamento indicaram menor propensão às disfunções e angústias sexuais, além de maiores chances de excitação durante a relação sexual (Dennerstein et al., 2006; Hayes et al., 2008; Thompson et al., 2011). Já as mulheres sexualmente ativas na pós-menopausa e que possuíam um relacionamento íntimo exibiram angústias e preocupações com os possíveis efeitos negativos das disfunções sexuais para o relacionamento, manutenção do interesse do parceiro e a frequência das relações sexuais (Dennerstein et al., 2006; Lonnèe-Hoffmann et al., 2014).

Em suma, a maioria dos estudos dessa categoria considera ser a menopausa uma das principais influências para as disfunções sexuais sem, contudo, ser isoladamente sua principal causadora. Assim, a menopausa parece ser o motivo das disfunções sexuais apenas quando seus sintomas são analisados em conjunto com outros fatores (relacionamentos afetivos, saúde física, função sexual pregressa e escolaridade). Por fim, há de se considerar que todos estes estudos também possuem limitações, pois as características das amostras, as diferenças culturais das mulheres participantes, os instrumentos e escalas quantitativas utilizadas e seu caráter transversal não permitem generalizações, sendo fundamental para isso uma análise mais ampla, longitudinal e que incorpore avaliações clínicas.

\section{Considerações Finais}

As evidências disponíveis nos estudos recuperados demonstram a complexidade que permeia as repercussões da menopausa para a sexualidade das mulheres, especialmente para as idosas. Este período da vida frequentemente associado ao envelhecimento é retratado por muitos estudos a partir das questões biológicas e patológicas que podem interferir na sexualidade devido às alterações hormonais e físicas. Assim, as hipóteses iniciais consideram a probabilidade de diminuição da lubrificação, da excitação, do desejo, do prazer e da satisfação sexual com o decorrer dos anos.

Apesar de essas dificuldades acometerem muitas mulheres, esses fatores não devem ser compreendidos isoladamente. Os resultados de grande parte dos estudos indicaram a importância da análise conjunta sobre os aspectos biológicos, psicológicos, sociais, histórico de vida, além da maneira como a sexualidade foi vivenciada ao longo da vida e a influência dos relacionamentos cultivados, bem como o papel dos padrões sociais, valores, mitos e tabus sobre sexualidade e a escolaridade das mulheres. Todos esses fatores estão diretamente relacionados à menopausa e podem representar distintas repercussões para a sexualidade.

Desse modo, não se constatou consenso entre os estudos, visto que a maioria abordou faixas etárias extensas e diversos contextos culturais, utilizando abordagens metodológicas distintas. A abordagem quantitativa se destacou e foi a mais utilizada, contribuindo para identificar estatisticamente as repercussões positivas (redescoberta do próprio corpo e da sexualidade; maior satisfação dos desejos sexuais; amadurecimento pessoal; melhorias na qualidade dos relacionamentos) e negativas (diminuição do desejo e do prazer sexual; dor no ato sexual; mudanças estéticas; dificuldades no funcionamento sexual em comparação a outros períodos da vida).

Além disso, algumas comparações puderam ser traçadas evidenciando diferenças entre a menopausa natural e a cirúrgica na vivência da sexualidade das mulheres. Destaca-se que a menopausa cirúrgica foi identificada em muitos estudos como causadora das repercussões negativas para a sexualidade. Já os estudos qualitativos, ao permitirem a maior expressão das mulheres participantes sobre o tema, favoreceram a exploração de outros fatores que influenciam a vivência da sexualidade no envelhecimento, tais como as experiências sexuais pregressas, as dúvidas, inseguranças, angústias e medos.

A respeito desta revisão, algumas limitações podem ser elencadas. Dentre elas pode-se considerar que o recorte temporal para a busca de artigos (limitado aos últimos dez anos) não permite uma análise aprofundada e historicamente orientada acerca da evolução dos estudos científicos na temática. Além disso, a utilização de apenas um juiz independente no processo de coleta de dados pode ser considerada fator limitante. Igualmente, 
a utilização apenas de artigos científicos (excluindo livros, capítulos, dissertações, teses e comunicações em eventos científicos dentre outros) também evidencia a parcialidade dos dados coletados sobre o objeto de investigação. Assim, essa revisão apresenta resultados significativos, porém delimitados, do tema em apreço.

Destaca-se também a necessidade de realização de outros estudos com metodologias mistas de coleta de dados para ampliar as informações sobre as possíveis repercussões da menopausa para a sexualidade e enriquecer a compreensão e a prática clínica sobre o tema. Ademais, estudos que tivessem como participantes apenas e exclusivamente mulheres idosas seriam importantes para preencher as lacunas observadas nesta revisão integrativa, haja vista que a maior parte dos estudos recuperados a partir da busca em bases de dados utilizou amostras com faixas etárias amplas (mulheres na pré e pós-menopausa, sem necessariamente serem idosas). Assim, novos estudos e investigações poderiam fornecer conhecimentos para as próprias mulheres idosas e para os profissionais de saúde, além de auxiliar no esclarecimento de possíveis dúvidas e sanar equívocos propagados pelo senso comum sobre a menopausa e a sexualidade no envelhecimento.

\section{Referências}

Almeida, L. H. R. B., Luz, M. H. B. A., \& Monteiro, C. F. S. (2007). Ser mulher no climatério: uma análise compreensiva pela enfermagem. Revista de Enfermagem da UERJ, 15(3), 370-375. Recuperado de http://www.facenf.uerj. $\mathrm{br} / \mathrm{v} 15 \mathrm{n} 3 / \mathrm{v} 15 \mathrm{n} 3 \mathrm{a} 08 . \mathrm{pdf}$

Alves, E. R. P., Costa, A. M., Bezerra, S. M. M. S., Nakano, A. M. S., Cavalcanti, A. M. T. S., \& Dias, M. D. (2015). Climacteric: intensity of symptoms and sexual performance. Texto Contexto Enfermagem, 24(1), 64-71. https://doi.org/10.1590/0104-07072015000590014

Araújo, I. A., Queiroz, A. B. A., Moura, M. A. V., \& Penna, L. H. G. (2013). Representações sociais da vida sexual de mulheres no climatério atendidas em serviços públicos de saúde. Texto Contexto Enfermagem, 22(1), 114-122. https://doi.org/10.1590/S0104-07072013000100014

Bandeira, L., Melo, H. P., \& Pinheiro, L. S. (2010). Mulheres em dados: o que informa a PNAD/IBGE, 2008. Revista do Observatório Brasil da Igualdade de Gênero, 1(1), 107-119.

Barreto, M., \& Heloani, R. (2011). Sexualidade e envelhecimento. In B. Trench \& T. E. C. Rosa (Orgs.). Nós e o outro: envelhecimento, reflexões, práticas e pesquisa (pp. 77-95). São Paulo, SP: Instituto de Saúde.

Biasus, F., Demantova, A., \& Camargo, B. V. (2011). Representações sociais do envelhecimento e da sexualidade para pessoas com mais de 50 anos. Temas em Psicologia, 19(1), 319-336. Recuperado de http://pepsic.bvsalud.org/ scielo.php?script=sci_arttext\&pid=S1413-389X2011000100025

Brasil. (2003). Estatuto do idoso. Brasília, DF: Ministério da Saúde.

Brasil. (2008). Ministério da Saúde, Secretaria de Atenção à Saúde. Manual de atenção à mulher no climatério/ menopausa. Brasília, DF: Secretaria de Atenção à Saúde.

Broome, M. E. (2000). Integrative literature reviews for the development of concepts. In B. L. Rodgers, \& K. A. Knafl (Orgs.), Concept development in nursing: foundations, techniques, and applications (pp. 231-250). Philadelphia, PA:W. B. Saunders.

Burri, A., Hilpert, P., \& Spector, T. (2015). Longitudinal evaluation of sexual function in a cohort of pre and postmenopausal women. The Journal of Sexual Medicine, 12(6), 1427-1435. https://doi.org/10.1111/jsm.12893

Butler, J. (2012). Problemas de gênero: feminismo e subversão da identidade. Rio de Janeiro, RJ: Civilização Brasileira.

Cabral, P. U. L., Canário, A. C. C., Spyrides, M. H. C., Uchôa, S. A. C., Eleutério Júnior, J., Amaral, R. L. G. et al. (2012). Influência dos sintomas climatéricos sobre a função sexual de mulheres de meia-idade. Revista Brasileira de Ginecologia e Obstetrícia, 34(7), 329-334. https://doi.org/10.1590/S0100-72032012000700007

Cavalcanti, I. F., Farias, P. N., Ithamar, L., Silva, V. M., \& Lemos, A. (2014). Função sexual e fatores associados à disfunção sexual em mulheres no climatério. Revista Brasileira de Ginecologia e Obstetrícia, 36(11), 497-502. https:// doi.org/10.1590/S0100-720320140004985 
Coelho, D. N. P., Daher, D. V., Santana, R. F., \& Santo, F. H. E. (2010). Percepção de Mulheres Idosas sobre Sexualidade: Implicações de Gênero e no Cuidado de Enfermagem. Revista da Rede de Enfermagem do Nordeste, 11(4), 163-173. Recuperado de http:/ /www.redalyc.org/articulo.oa?id=324027972017

Correa, M. F., Machado, J. U., Vega, S. V., Zambrano, N. B., Valbuena, G., \& Benítez, A. C. (2012). Respuesta sexual em mujeres posmenopáusicas. Revista de Obstetricia y Ginecología de Venezuela, 72(2), 103-114. Recuperado de http://www.scielo.org.ve/scielo.php?script=sci_arttext\&pid=S0048-77322012000200005

Costa,G. M.C., \&Gualda, D. M.R.(2008). Conhecimento e significado culturaldamenopausa paraumgrupodemulheres. Revista da Escola de Enfermagem da USP, 42(1), 81-89. https://doi.org/10.1590/S0080-62342008000100011

Davison, S. L., Bell, R. J., LaChina, M., Holden, S. L., \& Davis, S. R. (2009). The relationship between self-reported sexual satisfaction and general well-being in women. The Journal of Sexual Medicine, 6(10), 2690-2697. https://doi.org/10.1111/j.1743-6109.2009.01406.x

Debert, G., \& Brigeiro, M. (2012). Fronteiras de gênero e a sexualidade na velhice. Revista Brasileira de Ciências Sociais, 27(80), 37-54. https://doi.org/10.1590/S0102-69092012000300003

Dennerstein, L., Koochaki, P., Barton, I., \& Graziottin, A. (2006). Hypoactive sexual desire disorder in menopausal women: a survey of western european women. The Journal of Sexual Medicine, 3(2), 212-222. https://doi.org/10.1111/j.1743-6109.2006.00215.x

Erenel, A. S., Golbasi, Z., Kavlak, T., \& Dilbaz, S. (2015). Relationship between menopausal symptoms and sexual dysfunction among married Turkish women in 40-65 age group. International Journal of Nursing Practice, 21(5), 575-583. https://doi.org/10.1111/ijn.12309

Feltrin, R. B., \&Velho, L. (2014). Sexuality after menopause: ethnographic study in a brazilian hospital school. Sexuality Research and Social Policy, 11(1), 76-87. https://doi.org/10.1007/s13178-013-0133-6

Fernandes, M. G. M. (2009). Problematizando o corpo e a sexualidade de mulheres idosas: o olhar de gênero e geração. Revista de Enfermagem da UERJ, 17(3), 418-422. Recuperado de http://www.facenf.uerj.br/v17n3/v17n3a21.pdf

Fleury, H. J.,\& Abdo, C. H. N. (2015). Sexualidade da mulher idosa. Diagnóstico Tratamento, 20(3), 117-120. Recuperado de http://files.bvs.br/upload/S/1413-9979/2015/v20n3/a4902.pdf

Fonnegra, M. P. S., \& Bojacá, E. E. R. (2009). Sexualidad y menopausia: um estúdio en Bogotá (Colombia). Revista Colombiana de Psiquiatria, 38(1), 85-98. Recuperado de http://www.scielo.org.co/scielo.php?script=sci_abstract\&pid=S0034-74502009000100007

Frugoli, A., \& Magalhães-Júnior, C. A. O. (2011). A sexualidade na terceira idade na percepção de um grupo de idosas e indicações para a educação sexual. Arquivos de Ciências da Saúde UNIPAR, 15(1), 83-95. https://doi.org/10.25110/arqsaude.v15i1.2011.3696

Gradim, C. V. C., Sousa, A. M. M., \& Lobo, J. M. (2007). A prática sexual e o envelhecimento. Cogitare Enfermagem, 12(2), 204-213. https://doi.org/10.5380/ce.v12i2.9826

Graziottin, A. (2007). Prevalence and evaluation of sexual health problems - HSDD in Europe. The Journal of Sexual Medicine, 4 (suppl 3), 211-219. https://doi.org/10.1111/j.1743-6109.2007.00447.x

Graziottin, A., Koochaki, P. E., Rodenberg, C. A., \& Dennerstein, L. (2009). The prevalence of hypoactive sexual desire disorder in surgically menopausal women: an epidemiological study of women in four european countries. The Journal of Sexual Medicine, 6(8), 2143-2153. https://doi.org/10.1111/j.1743-6109.2009.01319.x

Hayes, R. D., Dennerstein, L., Bennett, C. M.,Sidat, M., Gurrin, L. C., \& Fairley, C. K. (2008). Risk factors for female sexual dysfunction in the general population: exploring factors associated with low sexual function and sexual distress. The Journal of Sexual Medicine, 5(7), 1681-1693. https:// doi.org/10.1111/j.1743-6109.2008.00838.x

Hinchliff, S., \& Gott, M. (2008). Challenging social myths and stereotypes of women and aging: heterosexual women talk about sex. Journal of Women \& Aging, 20(1/2), 65-81. https://doi.org/10.1300/J074v20n01_06

Instituto Brasileiro de Geografia e Estatística - IBGE. (2011). Sinopse do Censo Demográfico de 2010. Rio de Janeiro: Instituto Brasileiro de Geografia e Estatística; 2011. Recuperado em 28 de Agosto, 2015, de http://biblioteca.ibge. gov.br/visualizacao/livros/liv49230.pdf

Kisa, S., Zeynelog, S., \& Ozdemir, N. (2012). Examination of midlife women's attitudes toward menopause in Turkey. Nursing and Health Sciences, 14(2), 148-155. https://doi.org/10.1111/j.1442-2018.2011.00671.x 
Kokcu, A., Kurtoglu, E., Bildircin, D., Celik, H., Kaya, A., \& Alper, T. (2015). Does surgical menopause affect sexual performance differently from natural menopause? The Journal of Sexual Medicine, 12(6), 1407-1414. https:// doi.org/10.1111/jsm.12891

Lonnèe-Hoffmann, R. A. M., Dennerstein, L., Lehert, P., \& Szoeke, C. (2014). Sexual function in the late postmenopause: a decade of follow-up in a population-based cohort of australian women. The Journal of Sexual Medicine, 11(8), 2029-2038. https://doi.org/10.1111/jsm.12590

McCall, K., \& Meston, C. (2007). Differences between pre and postmenopausal women in cues for sexual desire. The Journal of Sexual Medicine, 4(2), 364-371. https://doi.org/10.1111/j.1743-6109.2006.00421.x

McCall-Hosenfeld, J. S., Jaramillo, S. A., Legault, C., Freund, K. M., Cochrane, B. B., Manson, J. et al. (2008). Correlates of sexual satisfaction among sexually active postmenopausal women in the women's health initiative-observational study. The Journal of General Internal Medicine, 23(12), 2000-2009. https://doi.org/10.1007/s11606-008-0820-9

Mendes, K. D. S., Silveira, R. C. C. P., \& Galvão, C. M. (2008). Revisão integrativa: método de pesquisa para a incorporação de evidências na saúde e na enfermagem. Texto \& Contexto Enfermagem, 17(4), 758-764. https://doi.org/10.1590/S0104-07072008000400018

Moraes, K. M., Vasconcelos, D. P., Silva, A. S., Silva, R. C., Santiago, L. M., \& Freitas, C. A. S. (2011). Companheirismo e sexualidade de casais na melhor idade: cuidando do casal idoso. Revista Brasileira de Geriatria e Gerontologia, 14(4), 787-798. https://doi.org/10.1590/S1809-98232011000400018

Nappi, R. E., \& Lachowsky, M. (2009). Menopause and sexuality: prevalence of symptoms and impact on quality of life. Maturitas, 63(2), 138-141. https://doi.org/10.1016/j.maturitas.2009.03.021

Oliveira, D. M., Jesus, M. C. P., \& Merighi, M. A. B. (2008). Climatério e sexualidade: a compreensão dessa interface por mulheres assistidas em grupo. Texto Contexto Enfermagem, 17(3), 519-526. https://doi.org/10.1590/S0104-07072008000300013

Polizer, A. A., \& Alves, T. M. B. (2009). Perfil da satisfação e função sexual de mulheres idosas. Fisioterapia em Movimento, 22(2), 151-158.

Santos, C. M. C., Pimenta, C. A. M., \& Nobre, M. R. C. (2007). A estratégia PICO para a construção da pergunta de pesquisa e busca de evidências. Revista Latino-americana de Enfermagem, 15(3), 508-511. https://doi.org/10.1590/S0104-11692007000300023

Santos, S. S. (2011). Sexualidade e Velhice. In E. V. Freitas \& L. Py (Orgs.), Tratado de Geriatria e Gerontologia. (3a ed., pp. 1543-1546). Rio de Janeiro, RJ: Guanabara Koogan.

Silva, G. M. D., Lima, S. M. R. R., \& Moraes, J. C. (2013). Avaliação da função sexual em mulheres após a menopausa portadoras de síndrome metabólica. Revista Brasileira de Ginecologia e Obstetrícia, 35(7), 301-308. https:// doi.org/10.1590/S0100-72032013000700004

Silva, K. G., \& Santiado, I. F. (2014). Um estudo sobre as relações de gênero e sexualidade no processo de feminização e envelhecimento da epidemia do HIV/AIDS. Qualit@s Revista Eletrônica, 15(2), 1-21. https://doi.org/10.18391/qualitas.v15i2.2221

Thompson, W. K., Charo, L., Vahia I. V., Depp, C., Allison, M., \& Jeste, D. V. (2011). Association between higher levels of sexual function, activity, and satisfaction and self-rated successful aging in older postmenopausal women. Journal of the American Geriatrics Society, 59(8), 1503-1508. https://doi.org/10.1111/j.1532-5415.2011.03495.x

Trench, B., \& Rosa, T. E. C. (2008). Menopausa, hormônios, envelhecimento: discursos de mulheres que vivem em um bairro na periferia da cidade de São Paulo, Estado de São Paulo, Brasil. Revista Brasileira de Saúde Materno Infantil, 8(2), 207-216. https://doi.org/10.1590/S1519-38292008000200008

Trindade, W. R., \& Ferreira, M. A. (2008). Sexualidade feminina: questões do cotidiano das mulheres. Texto Contexto Enfermagem, 17(3), 417-426. https://doi.org/10.1590/S0104-07072008000300002

Turato, E. R. (2008). Tratado de metodologia da pesquisa clínico-qualitativa: construção teórico epistemológica, discussão comparada e aplicação nas áreas de saúde e humanas. Petrópolis, RJ: Vozes.

Valadares, A. L. R., Machado, V.S. S., Costa-Paiva, L.,Osis, M.J., Sousa, M.H., \& Pinto-Neto, A. M. (2013). Self-perception of sexual life and associated factors: a population study conducted in women aged 50 or more years. Revista Brasileira de Ginecologia e Obstetrícia, 35(7), 295-300. https://doi.org/10.1590/S0100-72032013000700003 
Valadares, A. L. R., Pinto-Neto, A. M., Osis, M. J., Sousa, M. H.; Costa-Paiva, L., \& Conde, D. M. (2008). Prevalence of sexual dysfunction and its associated factors in women aged 40-65 years with 11 years or more of formal education: a population-based household survey. Clinics (São Paulo), 63(6), 775-782. https://doi.org/10.1590/S1807-59322008000600012

Valença, C.N., Nascimento Filho, J.M.N.,\&Germano, R.M. (2010). Mulherno climatério:reflexões sobredesejosexual, beleza e feminilidade. Saúde e Sociedade, 19(2), 273-285. https://doi.org/10.1590/S0104-12902010000200005

Vieira, K. F. L., Miranda, R. S., \& Coutinho, M. P. L. (2012). Sexualidade na velhice: um estudo de representações sociais. Psicologia e Saber Social, 1(1), 120-128. Recuperado de http://www.e-publicacoes.uerj.br/index.php/psi-sabersocial/article/view/3250/2257

Woloski-Wruble, A. C., Oliel, Y., Leefsma, M., \& Hochner-Celnikier, D. (2010). Sexual activities, sexual and life satisfaction, and successful aging in women. The Journal of Sexual Medicine, 7(7), 2401-2410. https://doi.org/10.1111/j.1743-6109.2010.01747.x

Wood, J. M., Mansfield, P. K., \& Koch, P. B. (2007). Negotiating sexual agency: postmenopausal women's meaning and experience ofsexualdesire. QualitativeHealthResearch, 17(2),189-200.https://doi.org/10.1177/1049732306297415

Yucel, C., \& Eroglu, K. (2013). Sexual problems in postmenopausal women and coping methods. Sexuality and Disability, 31(3), 217-228. https://doi.org/10.1007/s11195-013-9306-8

Zampieri, M. F. M., Tavares, C. M. A., Hames, M. L. C., Falcon, G. S., Silva, A. L., \& Gonçalves, L. T. (2009). O processo de viver e ser saudável das mulheres no climatério. Escola Anna Nery,13(2), 305-312. https://doi.org/10.1590/S1414-81452009000200010

\section{Izabella Lenza Crema}

Mestranda do Programa de Pós-Graduação em Psicologia (PPGP) - Universidade Federal do Triângulo Mineiro (UFTM), Uberaba - MG. Brasil.

E-mail: izabellalenza@gmail.com

\section{Rafael De Tilio}

Docente do Programa de Pós-Graduação em Psicologia (PPGP) - Universidade Federal do Triângulo Mineiro (UFTM), Uberaba - MG. Brasil.

E-mail: rafaeldetilio.uftm@gmail.com

\section{Maria Teresa de Assis Campos}

Mestranda do Programa de Pós-Graduação em Psicologia (PPGP) - Universidade Federal do Triângulo Mineiro (UFTM), Uberaba - MG. Brasil.

E-mail: mtassiscampos@hotmail.com

Endereço para envio de correspondência:

Universidade Federal do Triângulo Mineiro - Departamento de Psicologia. Avenida Getúlio Guaritá, 159, sala 320,

Bairro Abadia. CEP: 38025-440.

Uberaba - MG, Brasil.

Recebido 09/09/2016

Reformulação 25/05/2017

Aprovado 13/06/2017

Received 09/09/2016

Reformulated 05/25/2017

Approved 06/13/2017 
Recebido 09/09/2016

Reformulado 25/05/2017

Aceptado 13/06/2017

Como citar: Crema, I. L., De Tilio, R., \& Campos, M. T. A. (2017). Repercussões da menopausa para a sexualidade de idosas: revisão integrativa da literatura. Psicologia: Ciência e Profissão, 37(3), 753-769. https://doi.org/ 10.1590/1982-3703003422016

How to cite: Crema, I. L., De Tilio, R., \& Campos, M. T. A. (2017). Menopause effects for the sexuality of older adults: an integrative literature review. Psicologia: Ciência e Profissão, 37(3), 753-769. https://doi.org/ 10.1590/1982-3703003422016

Cómo citar: Crema, I. L., De Tilio, R., \& Campos, M. T. A. (2017). Efectos de la menopausia para la sexualidad: una revisión de literatura. Psicologia: Ciência e Profissão, 37(3), 753-769. https:/ /doi.org/ 10.1590/1982-3703003422016 